\title{
When less is more: mindfulness predicts adaptive affective responding to rejection via reduced prefrontal recruitment
}

\author{
Alexandra M. Martelli, ${ }^{1}$ David S. Chester, ${ }^{1}$ Kirk Warren Brown, ${ }^{1}$ \\ Naomi I. Eisenberger, ${ }^{2}$ and C. Nathan DeWall ${ }^{3}$
}

\author{
${ }^{1}$ Department of Psychology, Virginia Commonwealth University, Richmond, VA 23284, USA, ${ }^{2}$ Department of \\ Psychology, University of California Los Angeles, Los Angeles, CA 90095, USA and ${ }^{3}$ Department of Psychology, \\ University of Kentucky, Lexington, KY 40506, USA
}

Correspondence should be addressed to Alexandra M. Martelli, Department of Psychology, Virginia Commonwealth University, 806 W. Franklin St., Richmond, VA 23284, USA. E-mail: martellia@vcu.edu

\begin{abstract}
Social rejection is a distressing and painful event that many people must cope with on a frequent basis. Mindfulnessdefined here as a mental state of receptive attentiveness to internal and external stimuli as they arise, moment-to-moment-may buffer such social distress. However, little research indicates whether mindful individuals adaptively regulate the distress of rejection-or the neural mechanisms underlying this potential capacity. To fill these gaps in the literature, participants reported their trait mindfulness and then completed a social rejection paradigm (Cyberball) while undergoing functional magnetic resonance imaging. Approximately 1 hour after the rejection incident, participants reported their level of distress during rejection (i.e. social distress). Mindfulness was associated with less distress during rejection. This relation was mediated by lower activation in the left ventrolateral prefrontal cortex during the rejection incident, a brain region reliably associated with the inhibition of negative affect. Mindfulness was also correlated with less functional connectivity between the left ventrolateral prefrontal cortex and the bilateral amygdala and the dorsal anterior cingulate cortex, which play a critical role in the generation of social distress. Mindfulness may relate to effective coping with rejection by not overactivating top-down regulatory mechanisms, potentially resulting in more effective long-term emotion-regulation.
\end{abstract}

Key words: mindfulness; ventrolateral prefrontal cortex; social rejection; emotion-regulation; fMRI

\section{Introduction}

Having positive and lasting relationships is considered a fundamental need, and this motivation underlies a majority of interpersonal interactions (Baumeister \& Leary, 1995; Ryan \& Deci, 2017). Social rejection-which occurs when an individual is ignored or excluded from a social interaction-is a distressing and painful threat to this need to belong (Williams, 2009). Rejection-related distress can lead to numerous negative personal and interpersonal outcomes, such as depression and anxiety (Leary, 2010), increased health-relevant inflammatory response (Dickerson et al., 2009) and aggressive retaliatory behavior (Chester \& DeWall, 2017). These problematic outcomes necessitate research into how individuals can more adaptively respond to rejection.

A quality of consciousness termed mindfulness offers people a potential means through which they can effectively regulate their negative emotional responses (e.g. Arch \& Landy, 2015; 
Keng et al., 2013). The present research examined whether dispositionally mindful individuals would experience less distress arising from social rejection. Using functional brain imaging, we further examined the underlying neural mechanisms of this relationship, focusing on the recruitment of top-down regulatory resources in the lateral prefrontal cortex.

\section{Neural correlates of social rejection and the regulation of negative affect}

The distressing nature of social rejection is instantiated in neural pain circuitry, including the dorsal anterior cingulate cortex (dACC) and anterior insula (AI; Eisenberger, 2012). Juxtaposed against the distress-promoting role of the $\mathrm{AACC}$ and AI is the ventrolateral prefrontal cortex (VLPFC), which serves to inhibit the painful and distressing nature of instances of social rejection (Eisenberger et al., 2003; Chester \& DeWall, 2015). This social pain-suppressing role fits with the broader literature on the VLPFC, implicating it as a critical region in the downregulation of negative affect (e.g. Goldin et al., 2008). The VLPFC is able to achieve this adaptive tuning of negative affect by inhibiting activation of sub-cortical regions including the dACC, AI and amygdala (Wager et al., 2008). However, such VLPFC recruitment during distressing situations is not without its costs.

\section{The excessive recruitment model: emotion-regulation failure due to prefrontal taxation}

If the VLPFC serves to effectively regulate negative affect in a 'top-down' manner, should this brain region be recruited as much as possible? Classic neural models of self-regulation argued that affect-induced self-regulatory failure was, in large part, due to a lack of lateral PFC recruitment and its subsequent inhibition of the amygdala (Heatherton \& Wagner, 2011). However, more recent research has indicated that overactivation, rather than underactivation, of the VLPFC predicted selfregulatory failure in the wake of negative affect (Chester \& DeWall, 2015). In another study, greater VLPFC recruitment during rejection did not predict self-regulatory success after an exclusionary incident, but instead predicted self-regulatory failure (Chester \& DeWall, 2015). These findings support an excessive recruitment model of emotion regulation failure, where a disproportionate regulatory response in the VLPFC taxes the top-down inhibitory functions of this region and produces a subsequent refractory period in which individuals are less successful at self-regulation. The brain is not a muscle that becomes fatigued with use, but neurons do exhibit refractory periods after substantial excitation (Purves, 1975). Such VLPFC refraction may possibly serve as a biomarker of self-regulatory failure. If topdown recruitment during rejection undermines later regulatory outcomes, what forms of emotion regulation might effectively handle the distress of rejection without incurring such costs?

\section{Emotion regulation without top-down recruitment: the case for mindfulness}

A growing body of research suggests that mindfulness can effectively regulate emotional responses to evocative stimuli (Hölzel et al., 2011; Guendelman et al., 2017). The term mindfulness has multiple historically and culturally embedded meanings (Dreyfus, 2011) and various conceptualizations and operationalizations are used today (for review see Quaglia et al., 2015). However, mindfulness has been classically described as concerning a sustained, receptive attention to current internal and external stimuli (e.g. Anālayo, 2003; Brown \& Ryan, 2003; Quaglia et al., 2015). There is indication that mindfulness is effective in downregulating negative affect and does so differently than reappraisal, which is among the most commonly studied in effective emotion regulation strategies.

Reappraisal involves reinterpreting the meaning of an emotional stimulus, typically by appraising the evocative stimulus in an objective, detached manner (e.g. reappraising being ignored by a friend as due to his recent romantic breakup; Gross \& John, 2003). Considerable research indicates that this regulatory strategy effectively reduces negative affect (Webb et al., 2012; Cutuli, 2014) and does so in a top-down manner, wherein projections from ventrolateral and other prefrontal brain structures exert an inhibitory top-down influence on sub-cortical, affect-generative regions such as the amygdala (Davidson et al., 2000; Banks et al., 2007).

While there is some indication that mindful emotion regulation can operate in a top-down manner similar to reappraisal (Hölzel et al., 2011), a growing evidence base suggests that it does not require top-down processing; this has been seen in, for example, decreased activation in the VLPFC among mindfulness trainees and dispositionally mindful individuals when confronted with such stimuli (Hölzel et al., 2011; Chiesa et al., 2013; Guendelman et al., 2017). Consistent with this body of evidence, dispositional mindfulness has been associated with a reduced electrocortical signature of emotion regulation that arises, in part, from the left VLPFC (Brown et al., 2013; Liu et al., 2012). This reduced top-down recruitment may reflect a 'bottom-up' form of emotion regulation among those dispositionally inclined toward, or trained in, mindfulness (e.g. Hölzel et al., 2011). Such bottom-up processing may rely on the modification of implicit (rather than explicit) emotion regulation and emotion generation systems (Guendelman et al., 2017). Supportive evidence of this comes from studies of self-reported and trained mindfulness, wherein lower reactivity to aversive stimuli in subcortical regions (e.g. amygdala) has been observed (e.g. Creswell et al., 2007; Hölzel et al., 2011). Dampening early emotional reactivity may lessen the need for top-down inhibition of such reactivity. As such, 'more mindful individuals [may require] less regulatory resources to attenuate emotional arousal' (Lutz et al., 2014, p. 776; c.f. Modinos et al., 2010).

While there is a growing body of research on the salutary emotion regulatory consequences of mindfulness, there is little research examining whether this quality can specifically attenuate the emotional distress of social exclusion. Molet et al. (2013) found that very brief instruction in a focused attention form of mindfulness meditation predicted quicker recovery from ostracism-related distress, relative to an unfocused attention control condition. It is currently unknown whether a general disposition toward mindful states predicts altered levels of rejection-related distress. This is an important topic because efforts to buffer exclusion-related distress have been largely unsuccessful, with the exception of pharmacological, psychotropic or brain stimulation interventions (DeWall et al., 2010; Molet et al., 2013; Riva et al., 2015). Mindfulness is comparatively benign, and evidence suggests that people can learn to more frequently abide in mindful states-that is, to become more dispositionally (trait) mindful (Quaglia et al., 2015; Goleman \& Davidson, 2017).

\section{Present study}

The present research had three specific aims. First, it tested whether dispositionally mindful individuals would respond to 
social rejection with less distress. Second, it tested whether an attenuated VLPFC response helps to explain the role of mindfulness in reduced social distress. Third, it examined the functional connectivity profile of mindful individuals' neural response to rejection, focusing on connections with brain regions critical to the generation of distress (dACC, AI and amygdala). To accomplish our specific aims, participants self-reported on trait mindfulness and then completed a social rejection paradigm (Cyberball) while undergoing functional magnetic resonance imaging (fMRI). After approximately 1 hour, participants reported their distress during the rejection experience. This delay was due to the presence of several unrelated MRI scans that occurred after the rejection task.

\section{Methods}

\section{Participants}

Participants were 40 neurologically and psychologically healthy undergraduates who participated in the study for course credit and payment ${ }^{1}$. Participants were excluded if they met any of the following criteria: obesity (body mass index $>29$ ), claustrophobia, color blindness, pregnancy, use of psychoactive medication, a history of seizures or metal inside the body. One participant was excluded from analyses due to excessive movement in the scanner during the Cyberball task and was therefore excluded from all subsequent analyses. Data from the 39 remaining participants were submitted for analysis (18 females; age: $M=18.97$, s.d. $=1.32$ ).

\section{Materials}

Mindful attention awareness scale. Trait mindfulness was measured using this 15 -item self-report scale assessing receptive attention to, and awareness of present moment events and experiences (example item: 'I find it difficult to stay focused on what is going on in the present moment'). Items were rated from 1 (almost always) to 6 (almost never), where higher scores indicated a higher level of dispositional mindfulness. The Mindful Attention Awareness Scale (MAAS) has shown strong internal consistency and 4-week test-retest reliability and correlates positively with various indices of healthy emotion regulation (Brown \& Ryan, 2003; Quaglia et al., 2015). In the present study, MAAS showed excellent internal consistency (Cronbach $\alpha=0.87$ ).

Need threat scale. To measure social distress arising from exclusion in the Cyberball task, participants completed this 20-item self-report scale measuring threats to four basic 'needs' represented by the following four subscales: Belongingness, SelfEsteem, Control and Meaningful Existence. Each item was rated on a seven-point Likert scale from 1 (do not agree) to 7 (agree), where higher scores indicated a higher level of social distress. As per common practice, the subscale scores were aggregated into a single score for all analyses. This measure showed excellent internal consistency in the present study (Cronbach $\alpha=0.90$ ) (Van Beest \& Williams, 2006).

Suspicion probe. After all study procedures and just prior to debriefing, participants were administered a three-item

${ }^{1}$ Data from these participants' fMRI scans have been presented elsewhere (e.g. Chester et al., 2016), though no analyses in the present research are redundant with any published findings. suspicion probe to assess whether they believed the Cyberball manipulation ('What do you think this study was about?', 'Did anything about the study seem strange?' and 'Did you believe that you had a partner during the Cyberball task?').

\section{Procedure}

Pre-scan questionnaires. Participants arrived at the laboratory and completed a computerized battery of personality questionnaires which included a demographics questionnaire and the 15-item MAAS (Brown \& Ryan, 2003) measuring basic trait mindfulness. Participants were then scheduled to arrive at our MRI facility approximately 7 days after the questionnaire session and excused.

Social rejection task. Upon arrival at the MRI center, participants were screened for safety and comfort in the MRI scanner, led through a practice version of the computer tasks they would perform in the scanner and were then positioned in the scanner. While undergoing fMRI, participants were socially accepted then rejected via the Cyberball task (as in Williams et al., 2000; Chester \& DeWall, 2015). Cyberball was administered using a three block-design (60 s for blocks 1 and 2; 81 s for block 3). Prior to each block, participants were instructed to rest for $10 \mathrm{~s}$ and then saw a $2 \mathrm{~s}$ screen, which instructed them to 'get ready' for the next block. Participants received an equal amount of ball tosses as did the other 'players' (i.e. 33\%) throughout the first 2.5 blocks (i.e. $150 \mathrm{~s}$; acceptance condition). Then participants stopped receiving the ball for the last $51 \mathrm{~s}$ of the task (last part of block 2 and all of block 3; rejection condition).

Post-scan questionnaires. After a series of anatomical and unrelated functional scans (alcohol and social cue reactivity tasks), participants were removed from the scanner and completed a battery of questionnaires that included the 20-item Need Threat Scale, which measured participants' level of social distress due to Cyberball exclusion (Williams, 2009) as well as the three-item suspicion probe. These measures were completed roughly $45-$ $60 \mathrm{~min}$ after the social rejection manipulation.

\section{MRI data acquisition and preprocessing}

All MRI data were obtained using a 3.0-T Siemens Magnetom Trio scanner. Echo planar BOLD images were acquired with a T2*- weighted gradient across the entire brain with a 3D shim (matrix size $=64 \times 64$, field of view $=224 \mathrm{~mm}$, echo time $=28 \mathrm{~ms}$, repetition time $=2.5 \mathrm{~s}$, slice thickness $=3.5 \mathrm{~mm}, 40$ interleaved axial slices, flip angle $=90^{\circ}$ ). To allow for registration to native space, a coplanar T1-weighted MP-RAGE was also acquired from each participant ( $1 \mathrm{~mm}^{3}$ isotropic voxel size, echo time$=2.56 \mathrm{~ms}$, repetition time $=1.69 \mathrm{~s}$, flip angle $\left.=12^{\circ}\right)$. The Oxford Center for Functional MRI of the Brain (FMRIB)'s Software Library (FSL version 5.0.9) was used to conduct all preprocessing and fMRI analyses (Smith et al., 2004; Woolrich et al., 2009). Reconstructed functional volumes underwent head motion correction to the middle functional volume using FSL's MCFLIRT tool (Jenkinson et al., 2002). FSL's Brain Extraction Tool was then used to remove nonbrain tissue from all functional and structural volumes (Smith, 2002). After a series of data quality checks, functional volumes underwent slice-timing correction, pre-whitening, were smoothed with a $5-\mathrm{mm}$ full-width-halfmaximum Gaussian kernel and were high-pass filtered (100 s cutoff). 


\section{MRI data analysis: whole-brain regression analyses}

Preprocessed fMRI data from the Cyberball task were analyzed using a two-level general linear model approach.

First level (within-participants). First, each participant's BOLD signal was modeled with a fixed-effects analysis, which separately modeled acceptance and rejection blocks as regressors using a canonical double-gamma hemodynamic response function with a temporal derivative. 'Get Ready' screens and all six motion parameters were also included in the analysis as regressors-of-no-interest. Rest blocks were not explicitly modeled in this analysis, resulting in an implicit baseline comparison. A linear contrast then compared the two task conditions (rejection $>$ acceptance). Resulting contrast images from this analysis were first linearly registered to native space structural volumes and then spatially normalized to an MNI stereotaxic space template image using FSL's FLIRT tool (resampled into $2 \times 2 \times 2 \mathrm{~mm}^{3}$; Jenkinson \& Smith, 2001; Jenkinson et al., 2002).

Second level (between-participants). Each participant's contrast volumes from the first step were then fed into a group-level, mixed-effects Generalized Linear Model (GLM) that modeled participants' MAAS scores as a between-participants regressor. Group average maps were then created for the main effect of rejection > acceptance and for the correlation between MAAS scores and this rejection-related brain activity at each voxel across the entire brain. Cluster-based thresholding (Worsley, 2001; Heller et al., 2006) was applied to each of the group activation maps. Family-wise error correction based on Gaussian random field theory was then applied to each voxel across the entire brain (corrected threshold: $Z>2.3, P<0.05$ ). Multiple comparison corrections were then constrained to the left and right VLPFC. Left and right VLPFC masks were constructed from the Automated Anatomical Labeling (AAL) atlas using the opercular, orbital, and triangular portions of the left and right inferior frontal gyrus (Tzourio-Mazoyer et al., 2002). We repeated this analytic approach for dACC, AI and amygdala regions-ofinterest (ROIs). Specifically, ROIs of the AI and dACC were obtained from previous fMRI research on social pain (e.g. Chester et al., 2014). The AI ROIs used a caudal boundary of $y=8$ to correspond to the agranular insula. The dACC ROI used a rostral boundary of $y=33$ and a caudal boundary of $y=0$. Left and right amygdala ROI masks were obtained from the AAL atlas.

\section{MRI data analysis: psychophysiological interaction analyses}

To assess functional connectivity during rejection $>$ acceptance, a psychophysiological interaction (PPI) analysis was performed using the left and right VLPFC as seed regions. The VLPFC ROIs were planned to be either anatomical or functional, depending on the results of the whole-brain regression analyses. This took the form of another first level (within-participants) GLM with the addition of two new regressors to the previously described GLM: the mean-centered time course of VLPFC activity across the Cyberball task, and an interaction term multiplying the VLPFC time course by the rejection block regressor (i.e. the PPI regressor). Linear contrasts compared participants' implicit baseline against this PPI regressor (PPI $>$ baseline). Activation maps from this first level analysis were then fed into a second level (between-participants) whole-brain regression analysis that modeled mean-centered MAAS scores as a regressor. Group average maps were then created that modeled the
Table 1. Zero-order Correlations Between Mindfulness, VLPFC, Social distress, Amygdala, \& dACC

\begin{tabular}{llllll}
\hline & 1 & 2 & 3 & 4 & 5 \\
\hline 1. MAAS & & & & & \\
2. Ventrolateral PFC & $-0.53^{* * *}$ & & & & \\
3. Need threat scale & $-0.43^{* *}$ & $0.64^{* * *}$ & & & \\
4. Right amygdala & $-0.37^{*}$ & 0.30 & 0.07 & & \\
5. Left amygdala & $-0.44^{* *}$ & $0.42^{* *}$ & $0.27^{\dagger}$ & 0.16 & \\
6. dACC & $-0.34^{*}$ & $0.34^{*}$ & 0.19 & 0.25 & $0.44^{* *}$ \\
\hline
\end{tabular}

${ }^{\dagger} \mathrm{P}<0.10,{ }^{*} \mathrm{P}<0.05,{ }^{* *} \mathrm{P}<0.01,{ }^{* * *} \mathrm{P}<0.001$.

MAAS, mindful attention awareness scale; PFC, prefrontal cortex; dACC, dorsal anterior congulate cortex.

correlation between MAAS scores and this PPI regressor at each voxel across the entire brain. Family-wise error correction based on Gaussian random field theory was then applied across the entire brain (corrected threshold: $Z>2.3, P<0.05$ ). Multiple comparison correction was also constrained to the AACC, AI and bilateral amygdala using the ROI masks detailed previously.

\section{Results}

\section{Descriptive statistics}

No participants reported any suspicion of the true nature of Cyberball task during the suspicion probe. We calculated trait mindfulness and social distress by summing across each participant's corresponding responses. Trait mindfulness scores exhibited substantial variability across the scale's 15-105 range, $M=61.33$, s.d. $=12.74$, observed range $=37.00-90.00$. Social distress scores also showed substantial variance across the scale's 20-140 range, $M=70.87$, s.d. $=18.20$, observed range $=38.00$ 121.00 and were negatively correlated with trait mindfulness (see Table 1 for zero-order correlations among all self-report variables).

\section{Neuroimaging results}

Across the entire brain, there were no significant correlations between neural activation during social rejection (rejection > acceptance) and trait mindfulness scores. When analyses were constrained to left and right VLPFC ROIs, trait mindfulness was inversely related to activity in the left VLPFC (Figure 1; 219 voxels, peak $Z=-3.78$, peak MNI coordinates: $x=-48, y=30$, $z=10)$. Functional data from this main effect cluster of the left VLPFC were converted to units of percent signal change, averaged and extracted from each participant (Mumford, 2007). ROI analyses that focused on the AACC, bilateral AI and bilateral amygdala did not reveal any significant associations between rejection-related brain activity and mindfulness.

\section{Functional connectivity analyses}

Using the VLPFC cluster from the previous ROI analyses as a seed region, mindfulness was negatively associated with connectivity between the VLPFC and left amygdala (peak voxel: $Z=-3.94, P=0.004$, MNI coordinates $(x, y, z)=-16,-8,-20 ; 104$ contiguous voxels; Figure 1), right amygdala (peak voxel: $Z=-3.20, P=0.041$, MNI coordinates $(x, y, z)=24,2,-26 ; 36$ contiguous voxels; Figure 1), and dACC (peak voxel: $Z=-4.07$, $P=0.042$, MNI coordinates $(x, y, z)=6,12,28 ; 93$ contiguous voxels; Figures 1 and $2 A-D)$. No significant connectivity results were observed in the left or right AI. Together these findings 

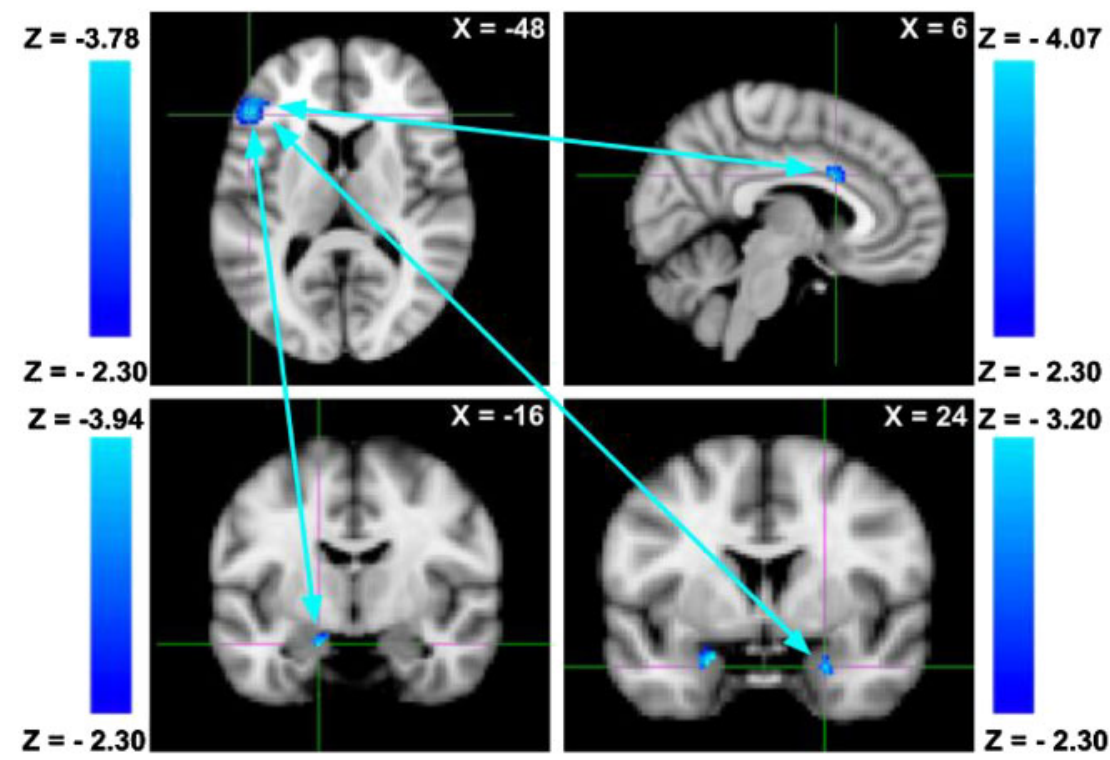

Fig. 1. Results of the VLPFC PPI and whole-brain regression analyses. Trait mindfulness was associated with less functional connectivity between VLPFC and both the bilateral amygdala and dACC. Coordinates are in MNI space.
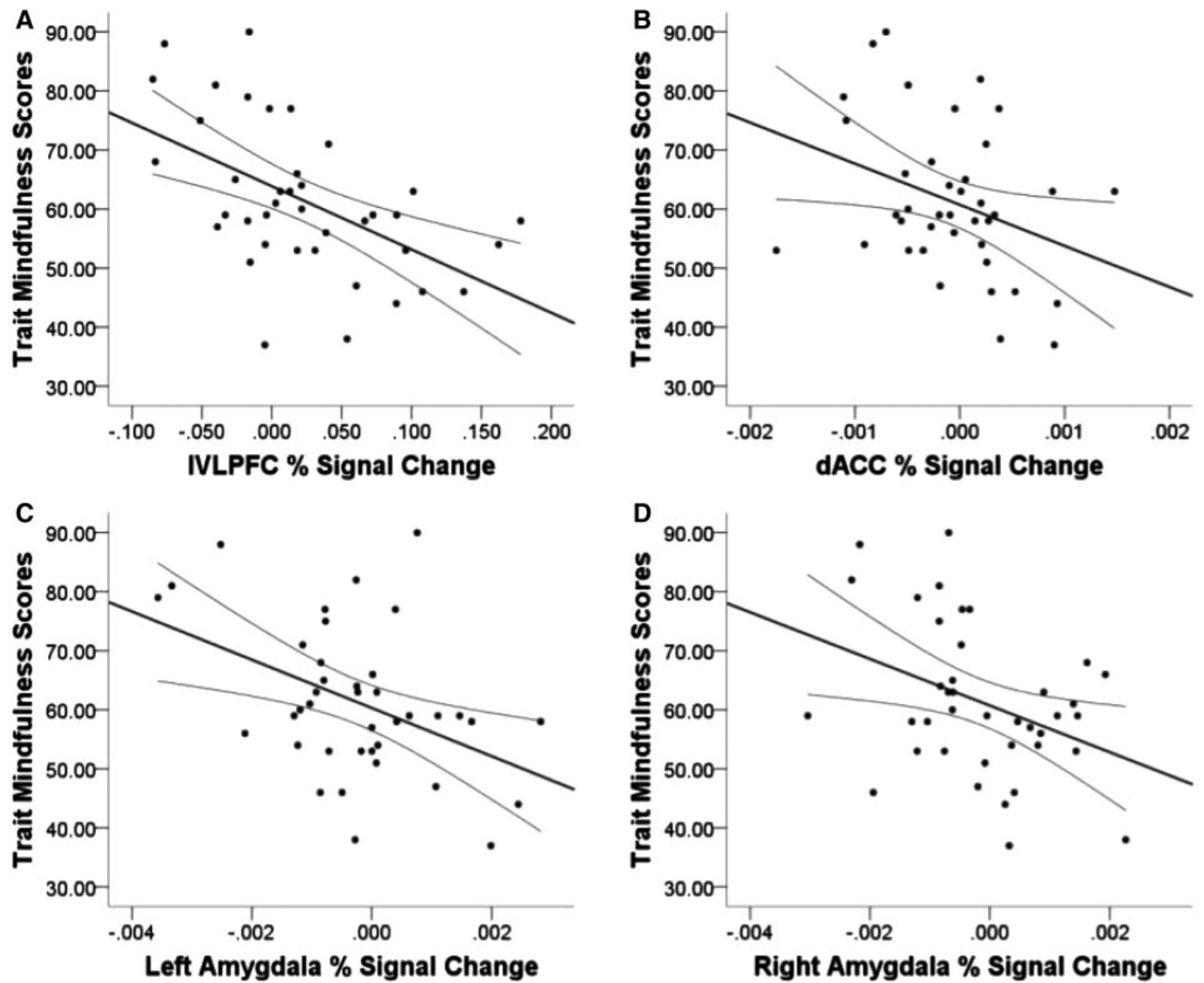

Fig. 2. (B) dACC, (C) left amygdala and (D) right amygdala functional connectivity estimates with the (A) left VLPFC during rejection > acceptance. 


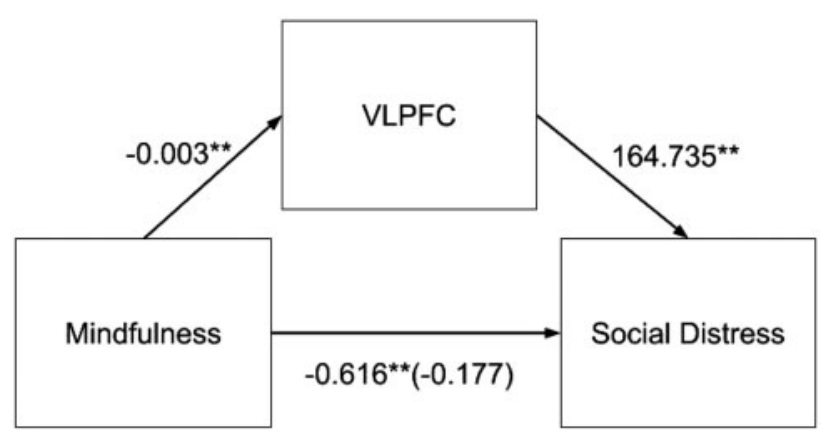

Fig. 3. Bootstrapped mediation model whereby less activation in VLPFC mediated the negative association between trait mindfulness and social distress. Parenthesized value represents the direct effect after controlling for the indirect effect (i.e. c' path). Values represent unstandardized regression coefficients. ${ }^{* *} \mathrm{P}<0.01$.

indicate that mindfulness was negatively correlated with functional connectivity between the left VLPFC and the dACC and bilateral amygdala during rejection. These PPI analyses were rerun replacing the rejection condition as the psychological variable with the acceptance condition, which the latter did not replicate these patterns of mindfulness-associated functional connectivity.

\section{Mediation analysis}

To assess whether rejection-related VLPFC activation mediated the relation between trait mindfulness and social distress, a bootstrapped-mediation model (using 5000 bias-corrected bootstrap samples; PROCESS macro, Model 4, Hayes, 2013) was fit, in which VLPFC activation was tested as a mediator between trait mindfulness and social distress. Results of this analysis showed that the negative correlation between trait mindfulness with social distress was mediated by decreased VLPFC activation during rejection $(B=-44, S E=0.16,95 \%$ confidence interval $[-0.82$, $-0.19]$; Figure 3 ). The resulting model explained $18.60 \%$ of the variance in social distress.

\section{Discussion}

Social rejection is often a distressing experience that can lead to adverse outcomes such as retaliatory aggression and risky sexual behavior (Kopetz et al., 2014; Chester \& DeWall, 2017). Therefore, it is important to investigate emotion regulation strategies that can reduce rejection-related distress. Mindfulness relates to a number of positive emotion regulation outcomes, such as quicker emotional recovery, reduced negative emotional responding, and reduced intensity of distress (Roemer et al., 2015). Therefore, mindfulness may represent one approach to emotion regulation that helps to reduce social distress. It has been argued that mindfulness is an inherent quality of mind (e.g. Brown \& Ryan, 2003; Kabat-Zinn, 2006), permitting tests of association between individual differences in basic trait mindfulness and the distress stemming from social rejection. The goals of the present study were to test whether dispositionally mindful individuals would respond to social rejection with less distress, and to begin to understand the neural mechanism(s) underlying this relation. Our study found that more mindful participants reported less social distress approximately an hour after being socially rejected. This is consistent with recent research finding that brief instruction in mindfulness predicted less distress after Cyberball-based social exclusion (Molet et al., 2013). The present results support the value of mindfulness as an effective means of managing rejection-related distress.

Regarding our second goal, we observed that an attenuated VLPFC response helped explain the association between mindfulness and reduced social distress. VLPFC activation during social rejection was inversely associated with trait mindfulness and this lower VLPFC activation mediated the link between mindfulness and social distress. These results indicate that mindful individuals' reduced prefrontal recruitment may be effective in regulating social distress, which is consistent with the idea that while greater recruitment of the VLPFC to regulate negative experiences can be adaptive, this is not always the case (Chester et al., 2016). Our findings fit this excessive inhibition framework that suggests over recruitment of prefrontal brain regions may not always be adaptive.

Our results are consistent with research on mindfulnessbased emotion regulation suggesting that regions implicated in bottom-up emotion regulation (e.g. amygdala \& dACC) and topdown emotion regulation (e.g. VLPFC) can function differently without the involvement of cognitive control (Guendelman et al., 2017). Indeed, mindfulness may act as a form of implicit emotion regulation, wherein individuals higher in trait mindfulness may be more effective regulators of their negative emotions because they are not overtaxing prefrontal regions implicated in emotion- and self-regulation. This line of research may have important implications for intervention models to employ strategies that do not overly tax individuals' prefrontal brain regions.

Additionally, the present study assessed the functional connectivity profile of mindful individuals' neural response to rejection and found three negatively correlated clusters in the left and right amygdala and dACC. These connectivity findings lend further support to our reverse inference that VLPFC activity during rejection serves to regulate brain regions critical to the generation of social distress (e.g. dACC; Eisenberger et al., 2003). Further, these findings fit well with the broader emotionregulation literature that implicate the VLPFC as an inhibitory hub of negative emotions that are generated in the amygdala and dACC (Wager et al., 2008; Heatherton \& Wagner, 2011).

Taken together, our findings are all consistent with the excessive inhibition model (Chester et al., 2016), which posits that over-recruitment of prefrontal regions may lead to subsequent failure to inhibit negative emotional experiences. Mindfulness, a well-established emotion-regulation approach, was able to achieve its salutary ends through less activation in VLPFC. This model challenges the prevailing paradigm in affective neuroscience, prompting further research into identifying the optimal (not too much or too little) extent of VLPFC recruitment that leads to adaptive emotional outcomes.

\section{Limitations and future directions}

There were several limitations that are important to mention. First, like all self-report measures of mindfulness, the MAAS taps a basic form of mindfulness (Brown et al., 2011). Most selfreport measures of mindfulness have been designed for respondents lacking mindfulness training, and thus the measures do not assess sophisticated expressions of it (Grossman, 2011). Yet the MAAS has been predicted adaptive emotion regulation operationalized at several levels of analysis (e.g. neurophysiological, subjective and behavioral) in normative and clinical populations (e.g. Quaglia et al., 2015; Hazlett-Stevens, 2017). That said, correlational research designs, such as that 
used here, leave predictive relations unclear, and variables correlated with mindfulness (e.g. neuroticism) may account for the neural and distress results reported here. Experimental research using mindfulness training trials is called for to better understand the neural underpinnings of mindful responses to social exclusion.

Another important limitation to consider involves participant selection and generalizability. Our sample consisted of undergraduate students enrolled in Psychology courses and future research should include community samples. Future research examining mindfulness, social distress and their neural associations could also be fruitfully extended into populations vulnerable to social exclusion and rejection (e.g. older lonely adults). Additionally, numerous safety restrictions are placed on individuals participating in fMRI research, which limits the generalizability of the results. Future research could invite ineligible participants to provide behavioral data and assess whether behavioral findings obtained in this subset of individuals are consistent with those obtained in a scanner environment.

A third limitation is that social distress was measured roughly 45-60 minutes following the exclusion experience. This approach may be problematic as the delay in response may introduce variance due to recovery, post-task emotion regulatory efforts, and other current psychological states. It is important to note that observing these lasting effects on emotion after a delay speaks to the robust nature of mindful emotionregulation. Future research should capture social distress at a prerejection baseline, immediately following the experience of rejection, and again after some time to see if these observed effects are replicated. Doing so will better capture the temporal dynamics of rejection-related distress, and the different roles that mindfulness plays at each of these time points, which may vary substantially.

Fourth, this study is limited due to its reliance upon the reverse inferences that activation in the VLPFC reflected dispositional mindfulness and that dACC and amygdala activation represented social distress. Inductive reasoning is problematic because these cognitive processes are not being directly tested, and these brain regions have many potential functions (Poldrack, 2011). Future studies may take advantage of techniques such as transcranial magnetic stimulation to assess whether less VLPFC activation during exclusion is in fact more adaptive.

\section{Conclusions}

The present study was an initial investigation into the neural mechanisms through which mindfulness attenuates the distress of social rejection. The results extend the growing body of research on mindful emotion regulation to show how this quality of mind may foster adaptive regulation of exclusion-related distress. More mindful individuals reported less distress after social exclusion and this relation was mediated by reduced VLPFC activation during that exclusion and showed less connectivity between the VLPFC and distress-generating regions of the dACC and amygdala. Not only do the current findings help to elucidate what underlying neural mechanisms are involved in the link between mindfulness and social distress, but they provide insight into the relation between mindfulness and the neural connectivity among specific brain regions during social rejection. The findings contribute to our understanding of brain function, mindfulness and their role in social rejection and have implications for the broader role of mindfulness in effectively regulating negative emotional experiences.

\section{Funding}

This experiment was funded by grants to the last author from the University of Kentucky's Center for Drug Abuse Research Translation (Sponsor: National Institute on Drug Abuse, Grant number: DA005312) and from the National Science Foundation (Grant number: BCS1104118). The content is solely the responsibility of the authors and does not necessarily represent the official views of any of the aforementioned sources of support.

Conflict of interest. None declared.

\section{References}

Anālayo, B. (2003). Satipatthāna: The Direct Path to Realization. Birmingham, England: Windhorse Publications.

Arch, J.J., Landy, L.N. (2015). Emotional benefits of mindfulness. Handbook of mindfulness: Theory, research, and practice, 208-24.

Baumeister, R.F., Leary, M.R. (1995). The need to belong: desire for interpersonal attachments as a fundamental human motivation. Psychological Bulletin, 117(3), 497-529.

Brown, K.W., Ryan, R.M. (2003). The benefits of being present: mindfulness and its role in psychological well-being. Journal of Personality and Social Psychology, 84(4), 822-48.

Brown, K.W., Goodman, R.J., Inzlicht, M. (2013). Dispositional mindfulness and the attenuation of neural responses to emotional stimuli. Social Cognitive and Affective Neuroscience, 8(1), 93-9.

Chester, D.S., DeWall, C.N. (2015). Prefrontal recruitment during social rejection predicts greater subsequent self-regulatory imbalance and impairment: neural and longitudinal evidence. NeuroImage, 101, 485-93.

Chester, D.S., DeWall, C.N. (2017). Combating the sting of rejection with the pleasure of revenge: A new look at how emotion shapes aggression. Journal of Personality and Social Psychology, 112(3), 413-30.

Chester, D.S., DeWall, C.N., Pond, R.S. (2016). The push of social pain: does rejection's sting motivate subsequent social reconnection? Cognitive, Affective, \& Behavioral Neuroscience, 16(3), 541-50.

Chester, D.S., Eisenberger, N.I., Pond, R.S., Richman, S.B., Bushman, B.J., DeWall, C.N. (2014). The interactive effect of social pain and executive functioning on aggression: an fMRI experiment. Social Cognitive and Affective Neuroscience, 9(5), 699-704.

Chester, D.S., Lynam, D.R., Milich, R., Powell, D.K., Andersen, A.H., DeWall, C.N. (2016). How do negative emotions impair self-control? A neural model of negative urgency. NeuroImage, 132, 43-50.

Chiesa, A., Serretti, A., Jakobsen, J.C. (2013). Mindfulness: top-down or bottom-up emotion regulation strategy? Clinical Psychology Review, 33(1), 82-96.

Creswell, J.D., Way, B.M., Eisenberger, N.I., Lieberman, M.D. (2007). Neural correlates of dispositional mindfulness during affect labeling. Psychosomatic Medicine, 69, 560-5.

Cutuli, D. (2014). Cognitive reappraisal and expressive suppression strategies role in the emotion regulation: an overview on their modulatory effects and neural correlates. Frontiers in Systems Neuroscience, 8, 1-6.

Davidson, R.J., Jackson, D.C., Kalin, N.H. (2000). Emotion, plasticity, context, and regulation: perspectives from affective neuroscience. Psychological Bulletin, 126(6), 591-4.

DeWall, C.N., MacDonald, G., Webster, G.D., et al. (2010). Acetaminophen reduces social pain: behavioral and neural evidence. Psychological Science, 21(7), 931-7. 
Dickerson, S.S., Gable, S.L., Irwin, M.R., Aziz, N., Kemeny, M.E. (2009). Social-evaluative threat and proinflammatory cytokine regulation: an experimental laboratory investigation. Psychological Science, 20(10), 1237-44.

Dreyfus, G. (2011). Is mindfulness present-centred and non-judgmental? A discussion of the cognitive dimensions of mindfulness. Contemporary Buddhism, 12(1), 41-54.

Eisenberger, N.I. (2012). The pain of social disconnection: examining the shared neural underpinnings of physical and social pain. Nature Reviews. Neuroscience, 13(6), 421.

Eisenberger, N.I., Lieberman, M.D., Williams, K.D. (2003). Does rejection hurt? An fMRI study of social exclusion. Science, 302(5643), 290-2.

Guendelman, S., Medeiros, S., Rampes, H. (2017). Mindfulness and emotion regulation: insights from neurobiological, psychological and clinical studies. Frontiers in Psychology, 8 (220), 1-23.

Goleman, D., Davidson, R. (2017). Altered Traits: Science Reveals How Meditation Changes Your Mind, Brain, and Body. New York: Penguin.

Gross, J.J., John, O.P. (2003). Individual differences in two emotion regulation processes: implications for affect, relationships, and well-being. Journal of Personality and Social Psychology, 85(2), 348-62.

Grossman, P. (2011). Defining mindfulness by how poorly I think I pay attention during everyday awareness and other intractable problems for psychology's (re)invention of mindfulness: comment on Brown. (2011). Psychological Assessment, 23(4), 1034-40.

Goldin, P.R., McRae, K., Ramel, W., Gross, J.J. (2008). The neural bases of emotion regulation: reappraisal and suppression of negative emotion. Biological Psychiatry, 63(6), 577-86.

Hayes, A.F. (2013). Introduction to Mediation, Moderation, and Conditional Process Analysis: A Regression-Based Approach. New York, NY: Guilford Press.

Hazlett-Stevens, H. (2017). Dispositional Mindfulness and Neural Correlates of Affect Regulation. Archives of Neuroscience, 4(3), 1-7.

Heatherton, T.F., Wagner, D.D. (2011). Cognitive neuroscience of self-regulation failure. Trends in Cognitive Sciences, 15(3), 132-9.

Heller, R., Stanley, D., Yekutieli, D., Rubin, N., Benjamini, Y. (2006). Cluster-based analysis of FMRI data. Neuroimage, 33(2), 599-608.

Hölzel, B.K., Lazar, S.W., Gard, T., Schuman-Olivier, Z., Vago, D.R., Ott, U. (2011). How does mindfulness meditation work? Proposing mechanisms of action from a conceptual and neural perspective. Perspectives on Psychological Science, 6(6), 537-59.

Jenkinson, M., Bannister, P., Brady, M., Smith, S. (2002). Improved optimization for the robust and accurate linear registration and motion correction of brain images. Neuroimage, 17(2), 825-41.

Jenkinson, M., Smith, S. (2001). A global optimisation method for robust affine registration of brain images. Medical Image Analysis, 5(2), 143-56.

Kabat-Zinn, J. (2006). Mindfulness-based interventions in context: past, present, and future. Clinical Psychology: Science and Practice, 10(2), 144-56.

Keng, S.L., Robins, C.J., Smoski, M.J., Dagenbach, J., Leary, M.R. (2013). Reappraisal and mindfulness: a comparison of subjective effects and cognitive costs. Behaviour Research and Therapy, 51(12), 899-904.

Kopetz, C., Pickover, A., Magidson, J.F., Richards, J.M., Iwamoto, D., Lejuez, C.W. (2014). Gender and social rejection as risk factors for engaging in risky sexual behavior among crack/cocaine users. Prevention Science, 15(3), 376-84.

Leary, M.R. (2010). Affiliation, acceptance, and belonging: the pursuit of interpersonal connection. Handbook of Social Psychology, 3(III), 24.
Liu, Y., Huang, H., McGinnis-Deweese, M., Keil, A., Ding, M. (2012). Neural substrate of the late positive potential in emotional processing. Journal of Neuroscience, 32(42), 14563-72.

Lutz, J., Herwig, U., Opialla, S., et al. (2014). Mindfulness and emotion regulation-an fMRI study. Social Cognitive and Affective Neuroscience, 9(6), 776-85.

Modinos, G., Ormel, J., Aleman, A. (2010). Individual differences in dispositional mindfulness and brain activity involved in reappraisal of emotion. Social Cognitive and Affective Neuroscience, 5(4), 369-77.

Molet, M., Macquet, B., Lefebvre, O., Williams, K.D. (2013). A focused attention intervention for coping with ostracism. Consciousness and Cognition, 22(4), 1262-70.

Mumford, J. (2007). A guide to calculating percent change with featquery. Unpublished Tech Report In: http://mumford. bol. ucla. edu/perchange_guide. pdf.

Poldrack, R.A. (2011). Inferring mental states from neuroimaging data: from reverse inference to large-scale decoding. Neuron, 72(5), 692-7.

Purves, D. (1975). Functional and structural changes in mammalian sympathetic neurones following interruption of their axons. The Journal of Physiology, 252(2), 429-63.

Quaglia, J.T., Brown, K.W., Lindsay, E.K., Creswell, J.D., Goodman, R.J. (2015). From conceptualization to operationalization of mindfulness. In Brown, K.W., Creswell, J.D., \& Ryan, R.M., editors. Handbook of Mindfulness: Theory, Research, and Practice, (pp. 179-99), New York: Guilford Press.

Riva, P., Romero Lauro, L.J., DeWall, C.N., Chester, D.S., Bushman, B.J. (2015). Reducing aggressive responses to social exclusion using transcranial direct current stimulation. Social Cognitive and Affective Neuroscience, 10(3), 352-6.

Roemer, L., Williston, S.K., Rollins, L.G. (2015). Mindfulness and emotion regulation. Current Opinion in Psychology, 3, 52-7.

Ryan, R.M., Deci, E.L. (2017). Self-Determination Theory. New York: Guilford Press.

Smith, S.M. (2002). Fast robust automated brain extraction. Human Brain Mapping, 17(3), 143-55.

Smith, S.M., Jenkinson, M., Woolrich, M.W., et al. (2004). Advances in functional and structural MR image analysis and implementation as FSL. Neuroimage, 23, S208-19.

Tzourio-Mazoyer, N., Landeau, B., Papathanassiou, D., et al. (2002). Automated anatomical labeling of activations in SPM using a macroscopic anatomical parcellation of the MNI MRI single-subject brain. Neuroimage, 15(1), 273-89.

Van Beest, I., Williams, K.D. (2006). When inclusion costs and ostracism pays, ostracism still hurts. Journal of Personality and Social Psychology, 91(5), 918-28.

Wager, T.D., Davidson, M.L., Hughes, B.L., Lindquist, M.A., Ochsner, K.N. (2008). Prefrontal-subcortical pathways mediating successful emotion regulation. Neuron, 59(6), 1037-50.

Webb, T.L., Miles, E., Sheeran, P. (2012). Dealing with feeling: a meta-analysis of the effectiveness of strategies derived from the process model of emotion regulation. Psychological Bulletin, 138(4), 775-808.

Williams, K.D. (2009). Ostracism: a temporal need-threat model. Advances in Experimental Social Psychology, 41, 275-314.

Williams, K.D., Cheung, C.K., Choi, W. (2000). Cyberostracism: effects of being ignored over the Internet. Journal of Personality and Social Psychology, 79(5), 748-62.

Woolrich, M.W., Jbabdi, S., Patenaude, B., et al. (2009). Bayesian analysis of neuroimaging data in FSL. NeuroImage, 45(1), S173-86.

Worsley, K.J. (2001). Statistical analysis of activation images. Functional MRI: An Introduction to Methods, 14, 251-70. 